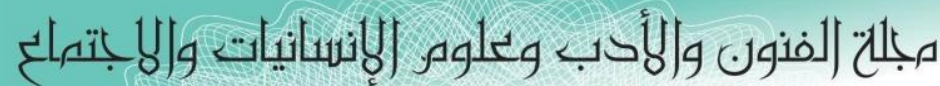
Journal of Arts, Literature, Humanities and Social Sciences

$\begin{array}{llc}\text { ISSN online: } 2414-3383 & \text { ديسمبر } 2019 \\ \text { ISSN print: } 2616-3810 & \text { Volume (46) } & \begin{array}{c}\text { (46) } \\ \text { December } 2019\end{array}\end{array}$

\title{
فاعلية الوسائط المتعددة في تحصيل طلاب الصف الثاني المتوسط واتجاهاتهم في مادة التاريخ العربي في الصفي الاسلامي في ماتهي
}

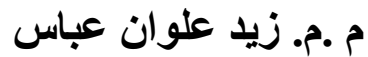



المديرية العامة لتربية القادسية - وزارة التربية ـ العراق

يهدف البحث الحالي الى معرفة فاعلية الوسائط المتعددة في تحصيل طلاب الصف الثاني المتوسط و اتجاهاتهم في مادة التاريخ العربي الاسلامي (الاجتماعيات) ولتحقيق ذلك اختار الباحثنان تصميماً ذا مجمو عتين متكافتتنين و اختار ا عثو ائياً عينة من طلاب الصف الثاني المتوسط في متوسطة حمور ابي التابعة لمركز محافظة القادسية للعام الدراسي(2018-2019) للفصل الثاني، تكونت عينة الدراسة من (60) طالباً بواقع (30) طالب لكل مجمو عة.



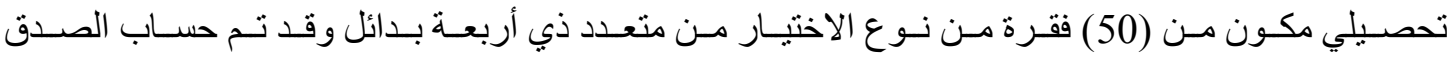

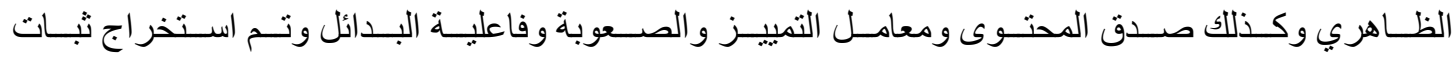



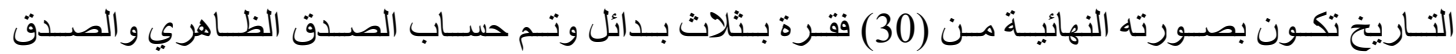



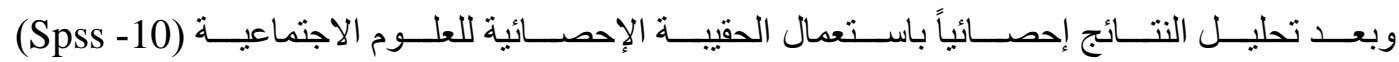


وفـق الوسـائط المتعـددة على طـلاب المجموعـة الضـابطة الـذين درسـو ا على وفـق الطريقـة الاعتياديـة في

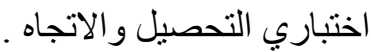




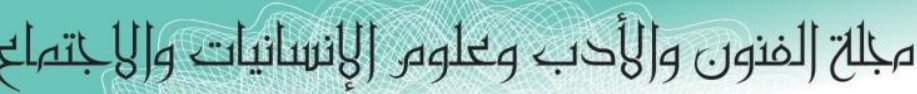

Journal of Arts, Literature, Humanities and Social Sciences

ISSN online: 2414 - 3383

ISSN print: 2616 - 3810

Volume (46)

(46) العدد

December 2019

\title{
The Effectiveness of Multimedia in the Achievement of Second Grade Students and their Attitudes in the Subject of Arab-Islamic History
}

\section{Zaid Alwan Abaas Alaa Hammed Mohsin}

\begin{abstract}
The current research aims to know the effectiveness of multimedia in the second grade students in the Mediterranean and attitudes in Arab and Islamic history to achieve this, the researcher chose two sovereign design randomly chose a sample of students in grade average in medium hammurabi of Al-Qadisiyah Province for the academic year (2018-2019) of chapter II, formed the study sample of (60) students at (30) Students per group.

With regard to research tools, the scholar to the preparation of the Scandisk and Defrag utilities initial research was to test a the achievement test (50) clause of the kind of choice of the four alternatives to the expense of the truth, as well as the sincerity of the content virtual labs and discrimination and difficulty and effectiveness of alternatives and the extraction of the test flat, where Alpha Cronbach (0.84) '(the second tool the scale was the trend toward the material of history, be the final form of the (30) clause three alternatives and the expense of honesty, truthfulness and virtual laboratories, and discrimination and difficulty of construction and the gauge either unchanged ‘(Alpha Cronbach ) unearthed in a manner (alpha) (0.88).

After analysis of the statistical results using statistical bag Social Sciences (Spss 10.) The Program (Microsoft Excel), the results showed the superiority of the experimental group students who studied in accordance with the multimedia to group students who studied the officer, according to the normal manner in the achievement test and the trend .
\end{abstract}


مبلحت (لفنون والأدب وعلوه الإنسانيات و|لهابتهماع Journal of Arts, Literature, Humanities and Social Sciences

ISSN online: 2414 - 3383

ISSN print: 2616 - 3810

\section{9 ديسمو \\ Volume (46)}

(46) العدد (2019

December 2019

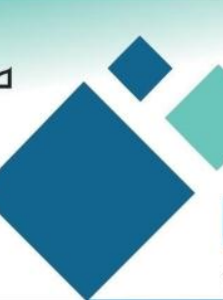



www.jalhss.com

\section{المبحث الاول \\ التعريف بالبحث}

مشكلة البحث:Problem of the Research

برزت مشكلة البحث الحالي في ضوء مأن ما يأتي:-

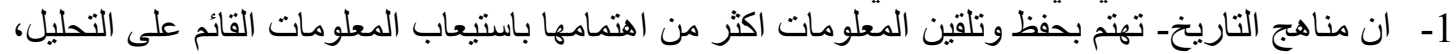

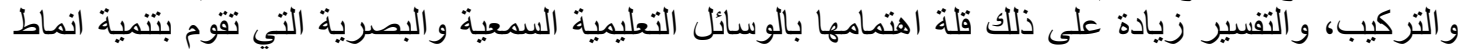

$$
\text { التفكير و الاتجاهات العلمية و اكساب المهار ات للطيلاب. }
$$







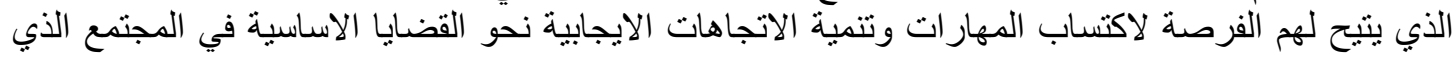



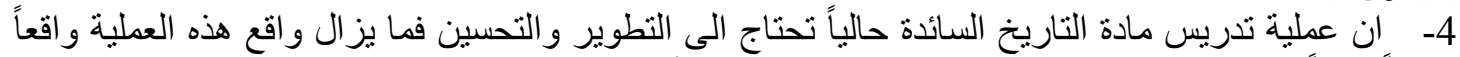

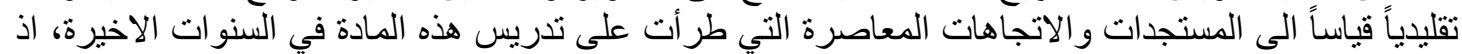

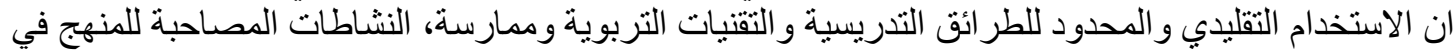
مجالات ضيقة جميعها لا تفيد في رفع مستوى التحصيل وتتمية الاتجاه وتطوير العملية التعليمية وتحقيق اهدافية التها



5- ان من الاهداف التربوية للمرحلة المتوسطة في مجال (النمو العقلي) هو تمكين الطلاب من اكتساب

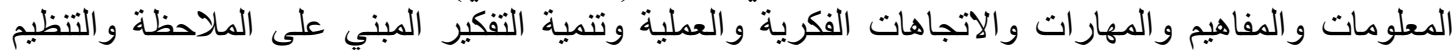

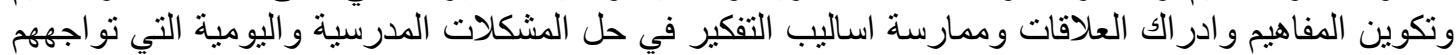
في المجتمع. ومن هنا تبرز مشكلة البحث الحالي بالسؤ ال التالي : (فاعلية الوسائط المنعددة في تحصيل طلاب الصفي التف الثاني المتوسط واتجاهاتهم في مادة التاريخ العربي

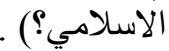

أهمية البحث: Importance of the Research

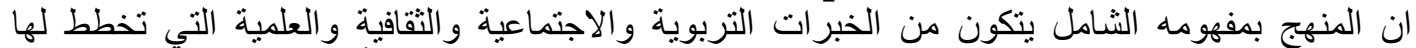

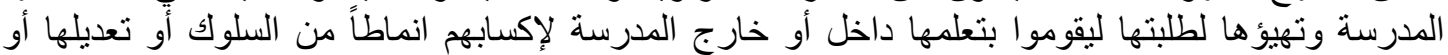

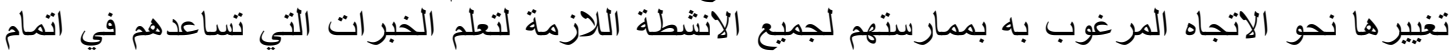

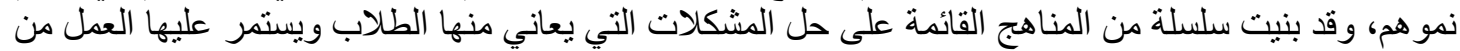



والادلة العلمية وتحقيق منطلبات العملية التربوية (Solomon\& Sue,2005:13)

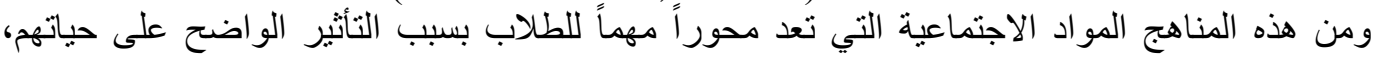

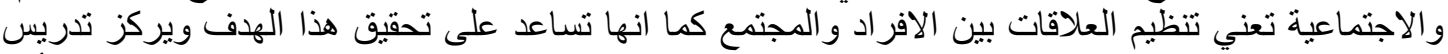



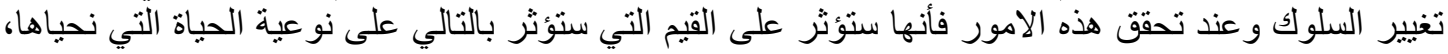

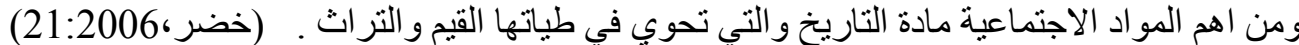

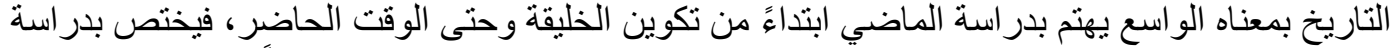







(كاتوت، (184:2009) 


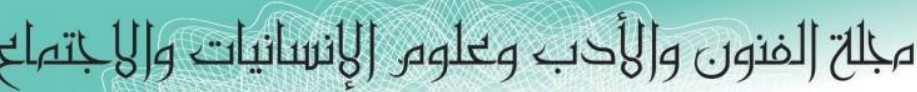

Journal of Arts, Literature, Humanities and Social Sciences

$\begin{array}{llc}\text { ISSN online: } 2414 \text { - } 3383 & \text { (46) } 2019 \text { ديسمبر } 2019 \\ \text { ISSN print: } 2616 \text { - } 3810 & \text { Volume (46) } & \text { December } 2019\end{array}$

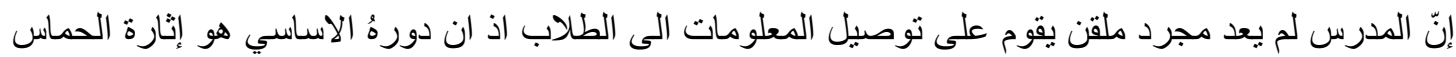

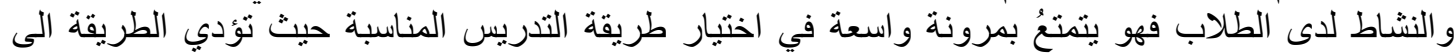

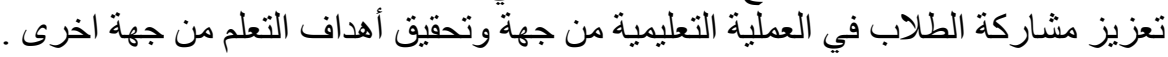







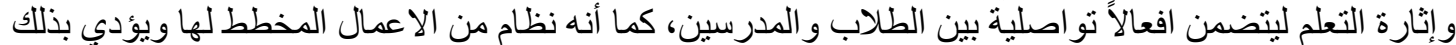

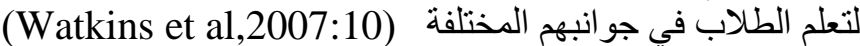
وتشيرُ الإحصائيات الى انخفاض فاعلية التعليم المدرسي وطر ائق التدريس في المرحلة الابتدائية و المنتوسطة

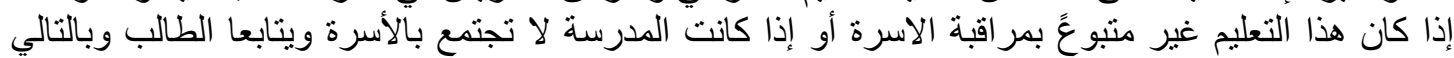

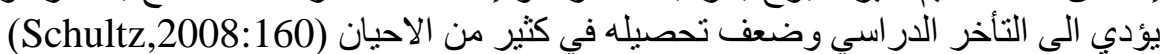

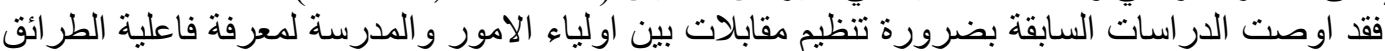
المستعملة في التدريس و الكثف المبكر عن القصور فيهاو المشاكل التي يعاني منها الطلاب في الصف الصف الدراسي (Evans, 2005:93)

ان التعلم بواسطة تقنيات تعليمية حديثة جعلت التعلم الناتج يكون عادة جيداً ومتقناً عن طريق ما تحدثة هذه التها







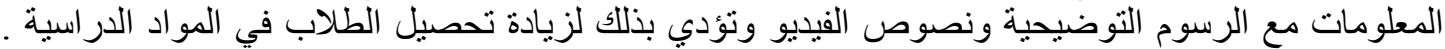

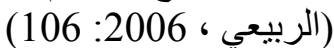

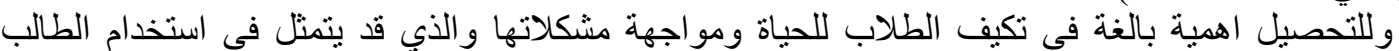

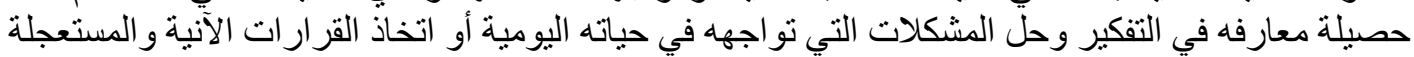

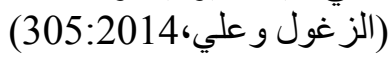

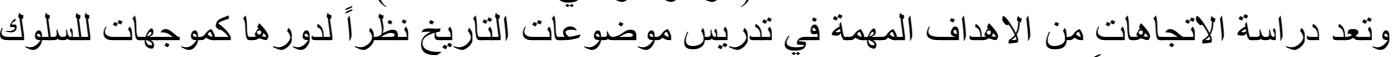



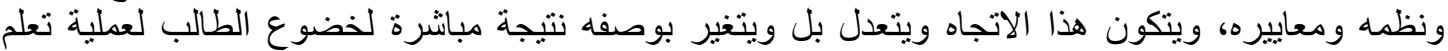
مباثر كما يحدث في المدرسة او بطريقة غير مبانرة كما يحدث عند احتكالك الطلبة بالبيئة التي يعيشون فيها.

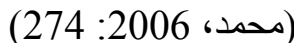

واستتاداً الى ما تقام تتجلى أهية إجراء هذا البحث بما يلي:

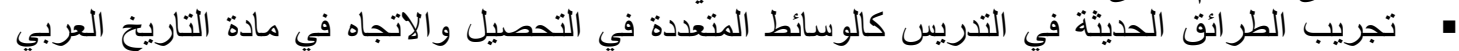

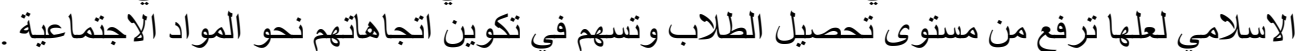

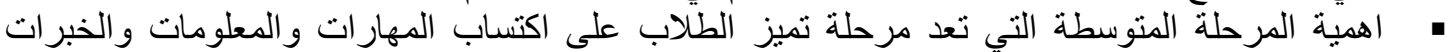

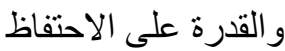
• أهمية مادة التاريخ العربي الاسلامي كونه يمثل جذر الامة الاسلامية و انه ليس علم الماضي فحسب بل علم

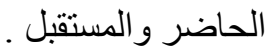

هاف البحث

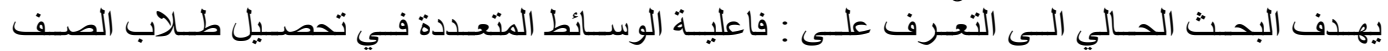
الثاني المنوسط و اتجاهاتهم في مادة التاريخ العربي الإسي الاسلامي.

فرضيتا البحث Hypotheses of Research


التجريبية الذين يدرسون بالوسائط المتعددة ومنوسط درجات طلاب المجموعة الضابطة الذين يدرسون بلات بالطريقة

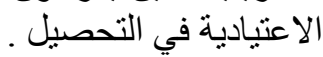
لا يوجد فروق ذات دلالة احصائية عند مستوى دلالة (0,05) بين متوسط درجات طلاب المجموعة 


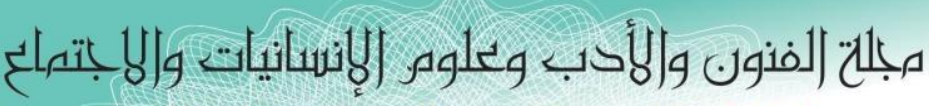

Journal of Arts, Literature, Humanities and Social Sciences

ISSN online: 2414 - 3383 ديسمبر 2019

ISSN print: 2616 - 3810

Volume (46)

العدد (46)

December 2019

LALHSS

WWw.jalhss.com

التجريبية الذين يدرسون الوسائط المتعددة ومتوسط درجات طلاب المجموعة الضابطة الذين يدرسون بالطريقة

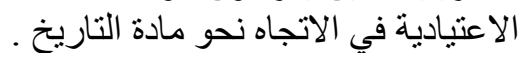

limitations of Research حدود البحث الانج

يقتصر البحث الحالي على:

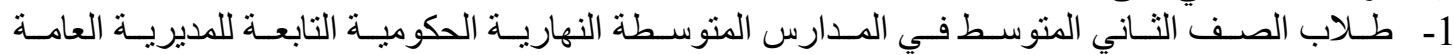

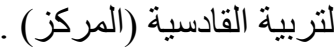




3- الفصل الدراسي الثاني من العام(2017-2018) ـ

Bounding of The Terms تحديد المصطلحات الوسائط المتعددة Multimedia: عرفها كل من:

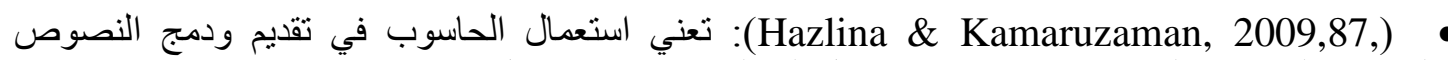
و الرسوم و الصوت و الفيديو برو ابط و ادوات تجعل المتعلم يندمج معها بشكل خلاق .



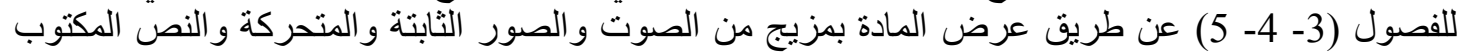
و المنطوق، و الفيديو، عن طريق جهاز عرض طرض البيانات (Data-Show) المدار و المسبطر عليه من قبل الباحثين.

التحصيل Achievement :عرفه : (Bedworth\&Albert,2010:7): بأنّه قياس درجة الكفاءة التي يحققها الطالب في بعض الإنها المهارات

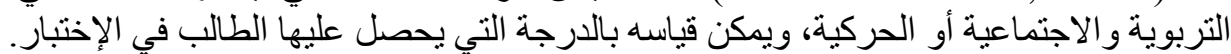



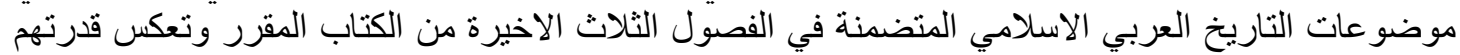

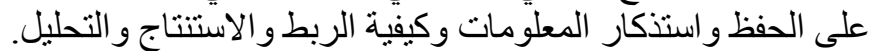
الاتجاه Attitude : عرفه:

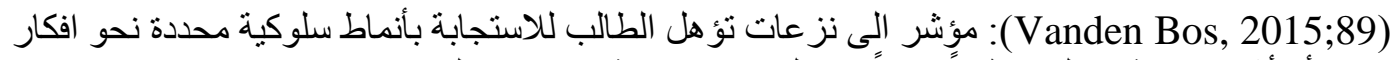

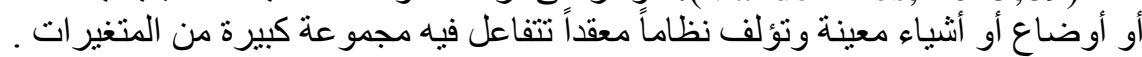

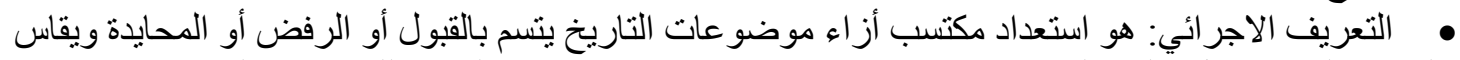


تاريخ للصف الثناني المتوسط .

$$
\text { المبحث الثانية }
$$

اولاً: التعليم الإكتروني E. Learning:

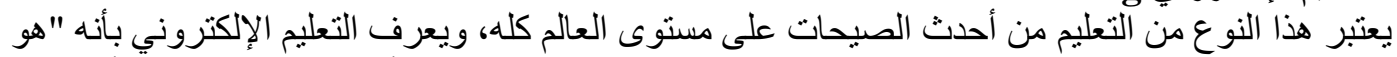


الإنترنت (الفار ، 2001، 2001 115).




ويعرف التعليم الإكتروني بأنه: نوع من أنواع التعليم عن بعد لاكتساب المهار التهات والمعارف بتفاعلات مدروسة 


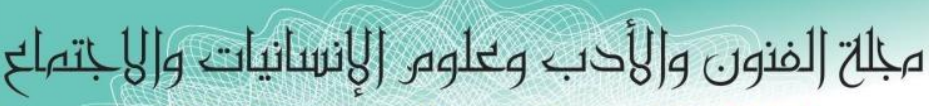
Journal of Arts, Literature, Humanities and Social Sciences



مع المو اد التعليمية التي يسهل الوصول إليها عن طريق استعمال بر امج مثل: بر امج التصفح . (Plack, 2002, 22)

Multi-Media الوسائط المتعددة

ساعدت تكنولوجيا الاتصال الحديثة على ظهور خدمات اتصالية جديدة أتاحتها هذه التكنولوجيا ولقد غيرت



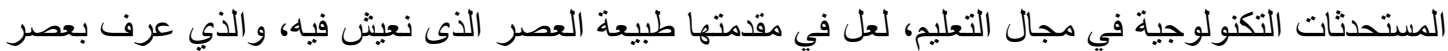

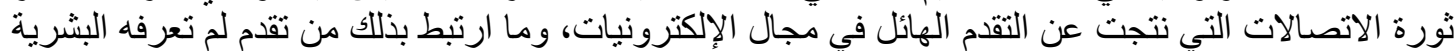

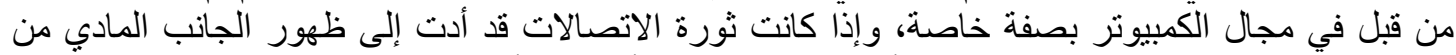

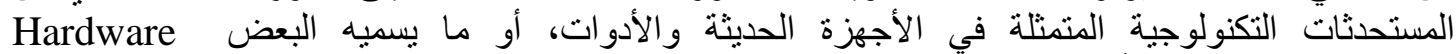
Revolution

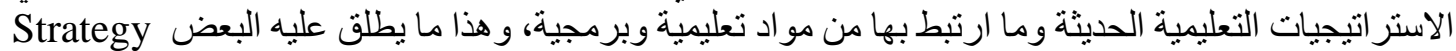
Software Revolution

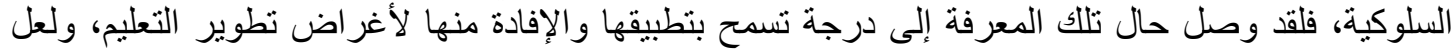

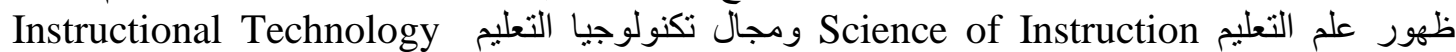
قد أوضحا إمكانية تطوير الممارسات التعليمية بصورة منهجية نظامية تسمح بزيادة فاعلية وكفاءة هذه

(العجرش، (18:2017)

المو اقف.

ما تتمتع بـه الوسائط المتعددة في التعليم

أ-التفاعلية Interactivity:

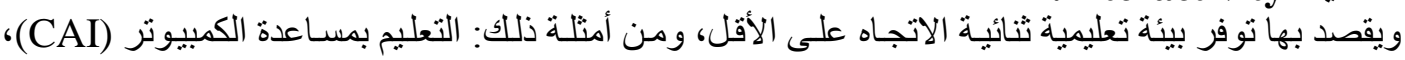



ب-الفردية Individuality حيث تتيح التعليم الفردي بما يناسب خصائص المتعلمين، ومن أمثلة ذلك: نظم التعليم بمساعدة الكمبيوتر. ج) حيث توفر بيئة تعلم متنو عـة البدائل بمـا يناسب خصنائص المتعلمين، وتثير قدر اتهم العقلية و المعرفية بتشكيلة مثير ات تخاطب حو اسهم المختلفة، ومن أمثلة ذللك: الوسائط المتعددة التفاعلية.

دالكونية Globality حيث تتيح للمتعلمين فرصة الانفتاح العالمي على مصادر التعلم في جميع أنحاء العالم، ومن أمثلة ذلك الإنترنت بخدماتها المختلفة.

هـالتكاملية Integrity

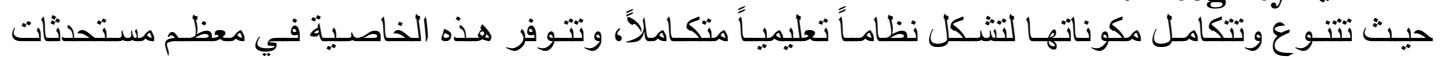



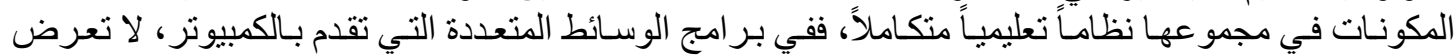

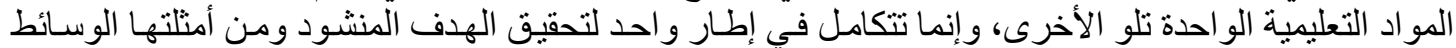

:Accessibility الإتاحدة

لابد و أن يكون هذا المستحدث متاحاً عندما يشعر المتعلم أنه في حاجـة إلى التعامل معـه بمعنى تلبيـة حاجاته من (زيتون، 2002، 134) التعلم.

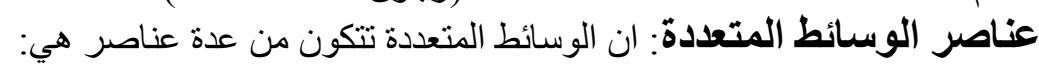

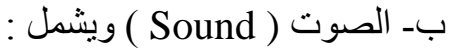



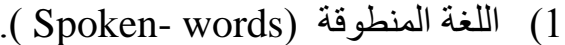

3)(المؤثرات الصوتية (Sound- effect).

(2) (1) الموسيقى (Music).

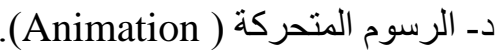

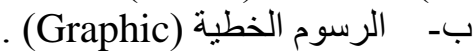
ج- الصور الثابتة (Still pictures) . 
مبلحت (لفنون والأدب وعلوه الإنسانيات و|لهاتهماع Journal of Arts, Literature, Humanities and Social Sciences

ISSN online: 2414 - 3383

ISSN print: 2616 - 3810

\section{9 ديسمبر \\ Volume (46)}

(46) (4016) (العدم)

December 2019

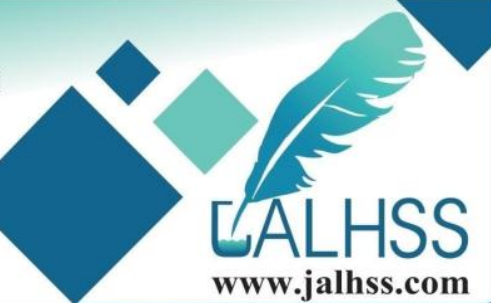

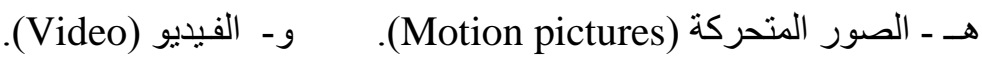

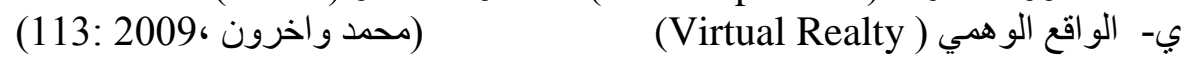

الفوائد التربوية للوسائط المتعددة: ان الفو ائد التربوية للوسائط المتعددة هي:

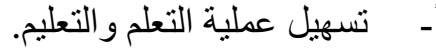
ب- ت توفير الجهذ و الوقت للمعلم و الطالب. ج- تثبيت التعلم و الاحتفاظ به باثشتر الك أكثر من حاسة في عملية التعلم.


بزملائه

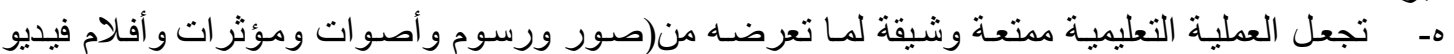
متحركة تنتد انتباه المتعلم). و - دعم عملية التعليم وتعزيز ها بعرض المعلومات بطر ائق متتو عة لمصادر المعرفة المختلفة.

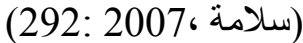

دور الوسائط التعليمية في تحسين عملية التعليم والتعلم:

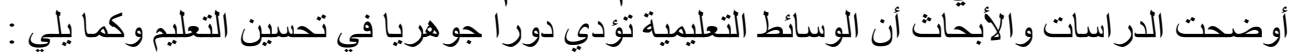

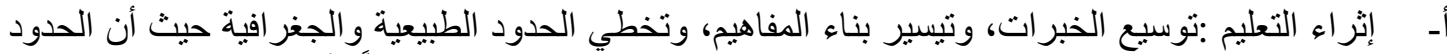

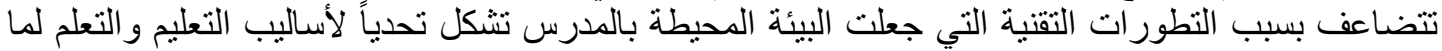

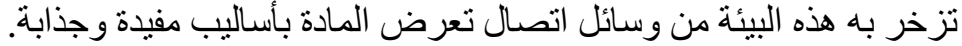

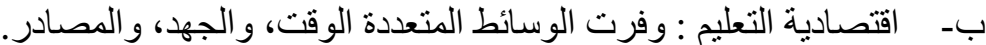

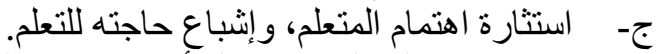
د- زيادة خبرة المتعلم ، و تجعله أكثر استعدادا للتعلم.

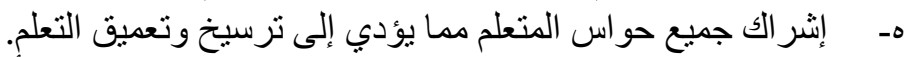

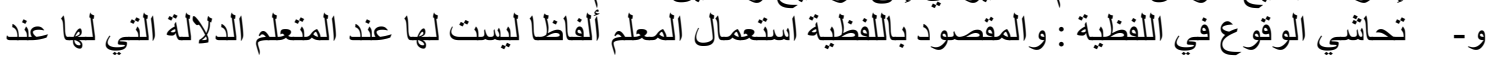

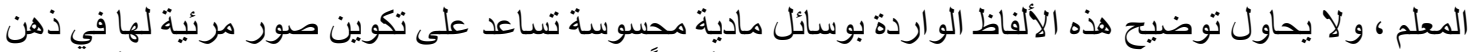

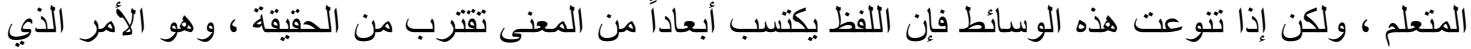



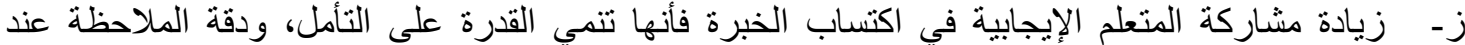



تكوين مفاهيم سليمة.



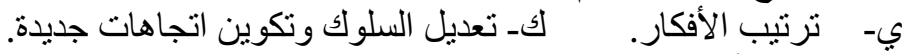

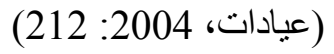

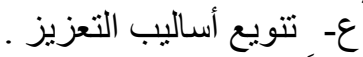
ثُانياً: التحصيل والاتجاه

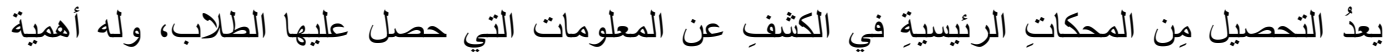

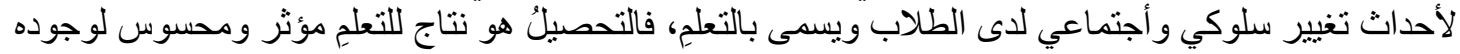

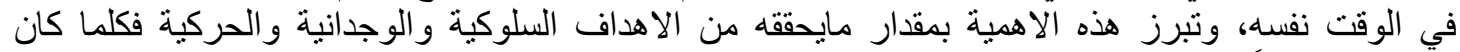

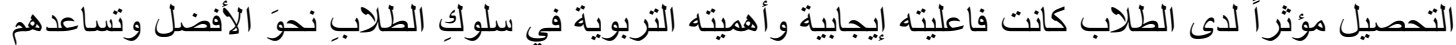

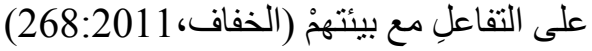

أن الطالب الذي يشعر باتجاهات ايجابية نحو المدرسة لا شك في أنها تعمل على تحرير طاقاته النفسية



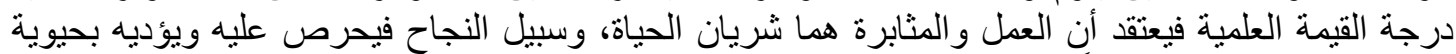






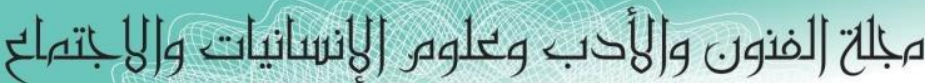

Journal of Arts, Literature, Humanities and Social Sciences

$\begin{array}{llc}\text { ISSN online: } 2414-3383 & \text { (46) } \\ \text { ISSN print: } 2616-3810 & \text { Volume (46) } & \begin{array}{c}\text { (46) } \\ \text { December } 2019\end{array}\end{array}$

ينظر إليه على أنه خبرة اكتسبها كي يستفيد منها في المستقبل (الخالدي، 2001، 165)

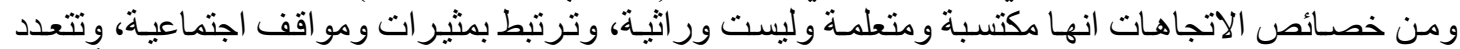

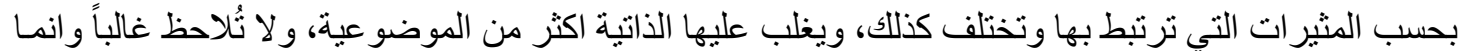






السلبية أبي المعارضة المطلقة. (المعايطة، 2000: 162)

من المسؤول عن اتجاهات الطلبة في مدارسنا :



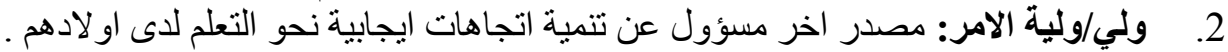

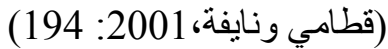

كيف يكشف المدرس عن الاتجاهات لاى طلبته :

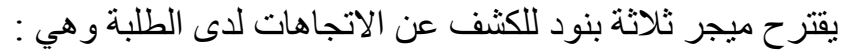



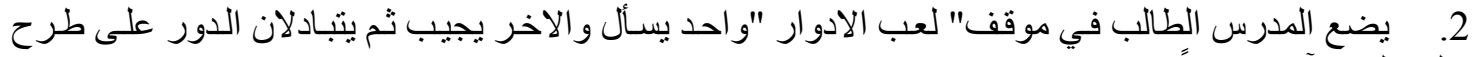

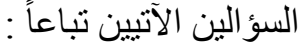

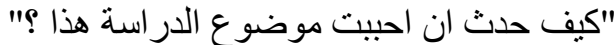

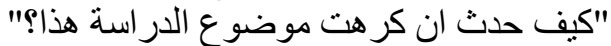
3. يستخدم المدرس دفتر الملاحظات لتسجيل أية استجابات بلاحظها على طلبته.



\section{المبحث الثالث}

\section{Procedures of the Research إجراءات البحث}



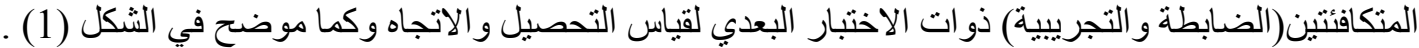

\begin{tabular}{|c|c|c|c|}
\hline المتغير التابع & المتغير المستقل & التكافؤ & المجموعة \\
\hline \multirow{2}{*}{ الاتجاه } & الوسائط المتعددة & \multirow{2}{*}{ 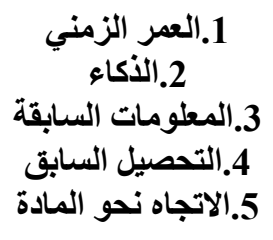 } & التجريبية \\
\hline & الطريقة الاعتيادية & & الضابطة \\
\hline
\end{tabular}

$$
\text { شكل (1) التصميم التجريبي لمجموعتي البحث }
$$

مجتمع البحث وعينته Research Sample and Population تحدد مجتمع البحث الحالي بطلاب الصف التهات الثاني المتوسط في المدارس الثانوية والمتوسطة الحكومية (الدراسة الصباحية) في مركز محافظة القادسية للعام الدراسي (2017- 2018)، واختار الباحثان عشوائياً 


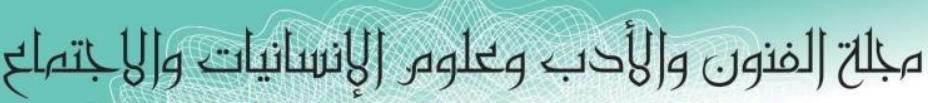
Journal of Arts, Literature, Humanities and Social Sciences

\begin{tabular}{|c|c|c|}
\hline $\begin{array}{l}\text { ISSN online: } 2414 \text { - } 3383 \\
\text { ISSN print: } 2616 \text { - } 3810\end{array}$ & ديسمبر (46) 2019 & $\begin{array}{c}\text { العدد (46) } \\
\text { December } 2019\end{array}$ \\
\hline
\end{tabular}

(بالقرعة) منوسطة حمور ابي من بين الددارس، والتي تحتوي على ثلاث شعب وبالتعيين العشو ائي اختار منها

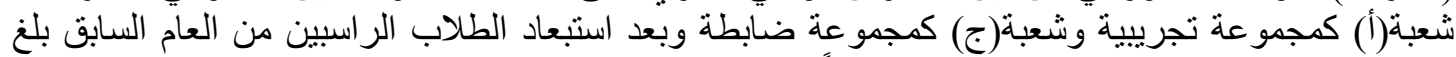

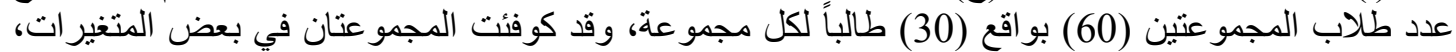



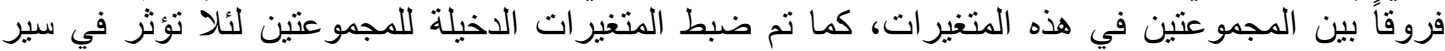
التجربة . مزونان.

مستلزمات البحث: Research Procedures

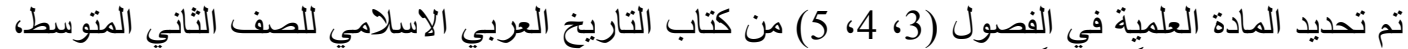

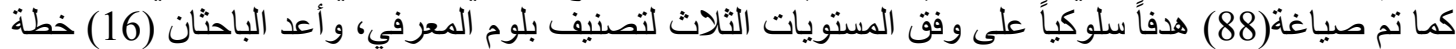

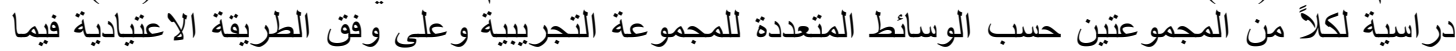

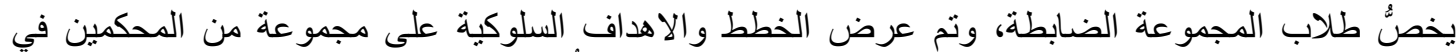

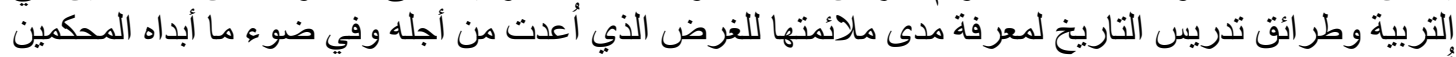



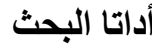
أعد الباحثان أداتين لقياس المتغيرات (التحصيل، الاتجاه) لمعرفة مدى تأثير المتغير المئنير المستقل (الوسائط

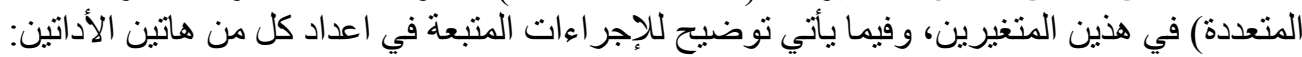

اولاً: اعداد الاختبار التحصيلي: Making Achievement test



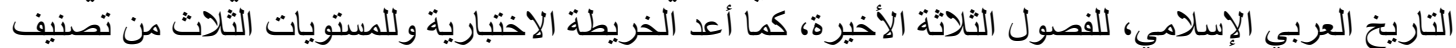

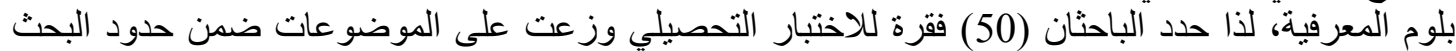

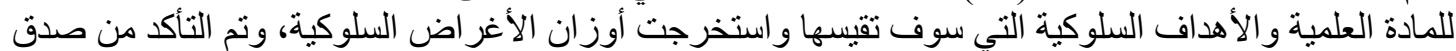

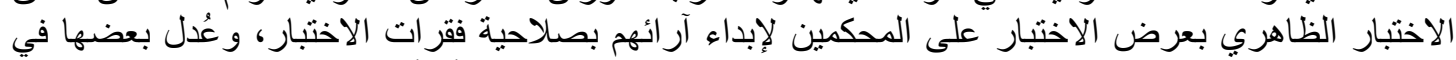



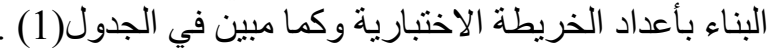
جذول (1) جلول المواصفات للاختبار التحصيلي

\begin{tabular}{|c|c|c|c|c|c|c|}
\hline \multirow{2}{*}{ الفقرات } & \multicolumn{3}{|c|}{ مستوى الأغراض السلوكية } & \multirow{2}{*}{ للمحتوى الوزن } & \multirow[b]{2}{*}{ الحصص } & \multirow{2}{*}{ التعليمي } \\
\hline & التطبيق & الفهج & $\begin{array}{l}\text { التذكر\% } \\
\% 49\end{array}$ & & & \\
\hline 18 & 3 & 5 & 10 & $\% 31$ & 6 & الثالث \\
\hline 22 & 2 & 8 & 12 & $\% 44$ & 7 &  \\
\hline 10 & 1 & 4 & 5 & $\% 25$ & 3 & الخامس \\
\hline 50 & 6 & 17 & 27 & $\% 100$ & 16 & المجموع \\
\hline
\end{tabular}

التطبيق الاستطلاعيّ للاختبار: اجرى الباحثان التجربة الاستطلاعية الأولية للاختبار على عبنة مكونة من (30)

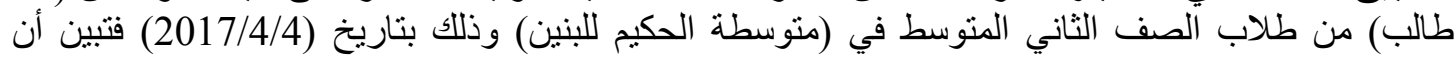



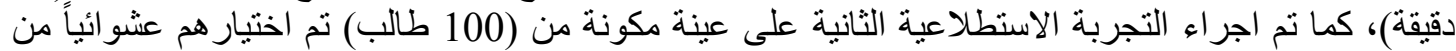
طلاب الصف الثاني المتوسط في (منوسطة الجزائر للبنين) في يوم الاربعاء المصادفة (2017/4/6) و أثرف 


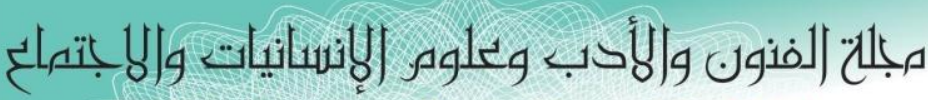

Journal of Arts, Literature, Humanities and Social Sciences

ISSN online: 2414 - 3383

ISSN print: 2616 - 3810

\section{9 ديسمبر \\ Volume (46)}

(46) العدد

December 2019

\section{¿ALHSS}

www.jalhss.com

الباحثان بنفسه على التطبيق، وبعد حساب منوسط الوقت المستغرق تبيّن أن الزمن المناسب لإتمام الإجابة هو



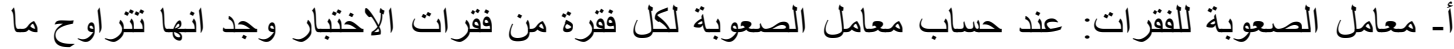





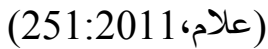

بـ معامل التمبيز للفقرات: عند حساب معامل التمبيز لكل فقرة من فقر ات الاختبار وجدها بين (0, إذ يرى إييل (Eble) أن فقرات الاختبار تعد جيدة إذا كانت قوة تمييزها (30)، 0.

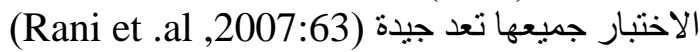

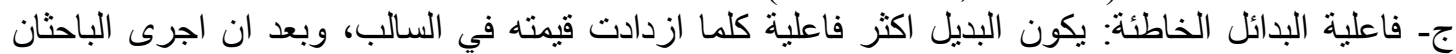

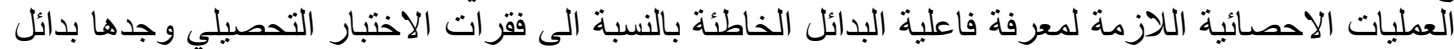

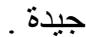
ثبات الاختبار : من أجل التحقق من ثبات الاختبار التحصيليّ، فقد استخدم الباحثان طريقة (الفا كرونباخ) إذ بلـغ

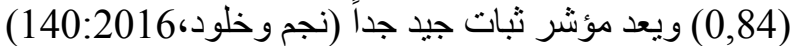

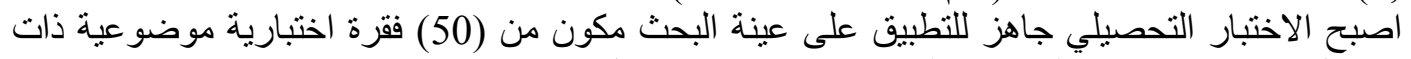





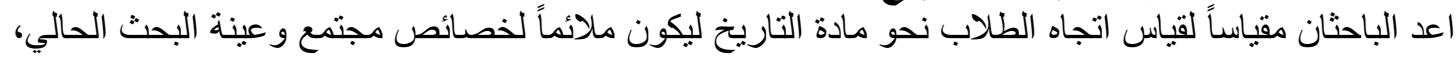



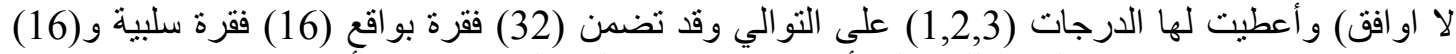

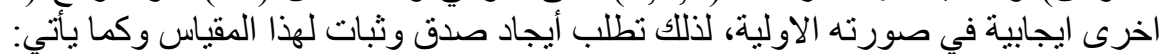

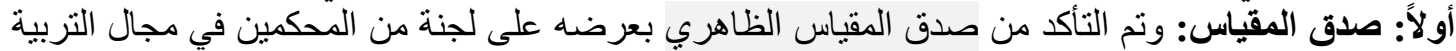


تحصلا على النسبة المطلوبة ونم الإبقاء على الفقرات التي حصلت على التى نسبة اتفاق (80\%) أو ألى أكثر، كما تم التأكد من الصدق البناء للمقياس بتحديد الخصائص الإئاء السايكومترية وكما يأتي :





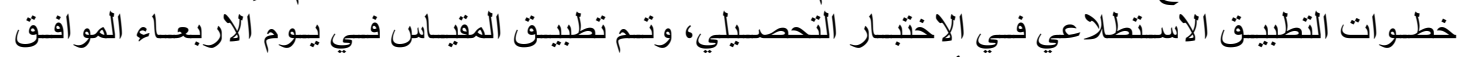



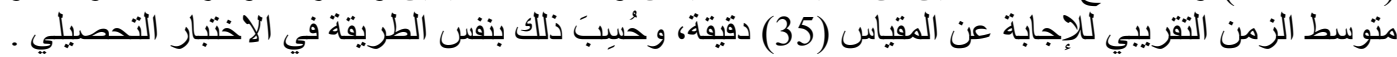

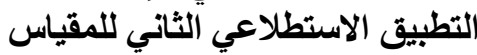

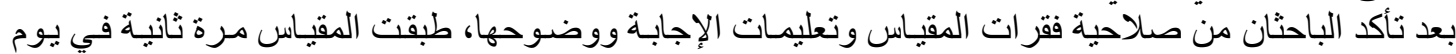

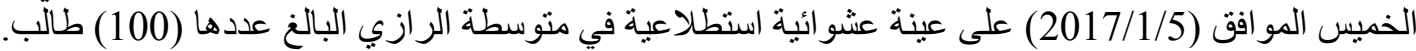



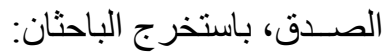



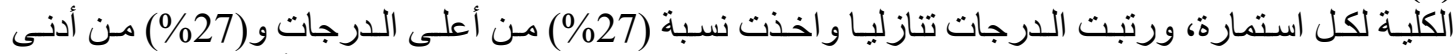



حسب الباحثان منوسطوقت الإجابة عن فقرات الاختبار باستعمال المعادلة الآتية : زمن الطالب الأول + زمن الطالب الثاني + زمن الطالب الثالث + زمن الطالب الر ابع + ... + زمن الطالب الطالب الأخير

عدد الطلاب الكلي 



Journal of Arts, Literature, Humanities and Social Sciences

$\begin{array}{llc}\text { ISSN online: } 2414-3383 & \text { ديسمبر } 2019 \\ \text { ISSN print: } 2616-3810 & \text { Volume (46) } & \begin{array}{c}\text { (46) } \\ \text { December } 2019\end{array}\end{array}$

لفقر ات المقياس تتر اوح ما بين (58,2,21-50)، و هي أعلى من القيمة الجدولية البالغة (1,09) عند مستوى دلالية

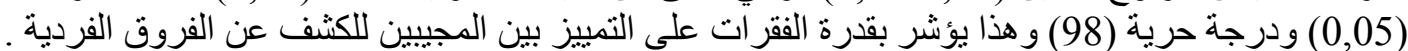



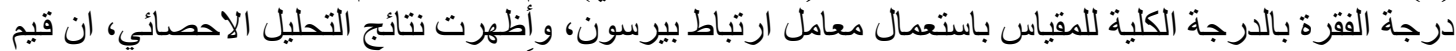



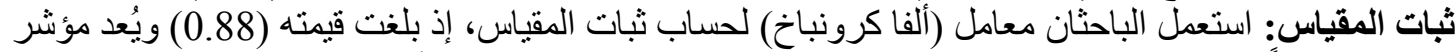

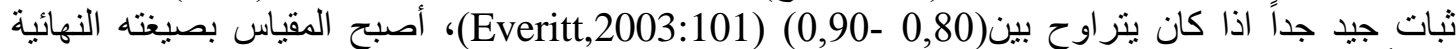

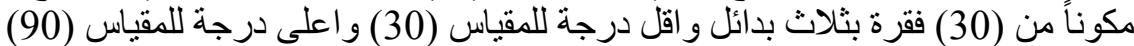

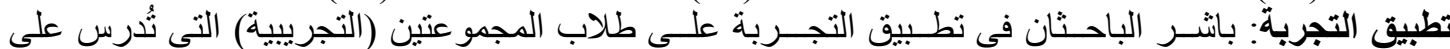





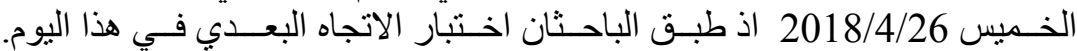

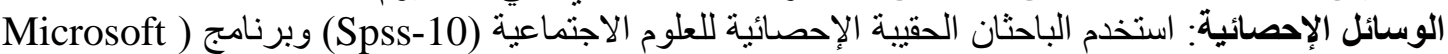

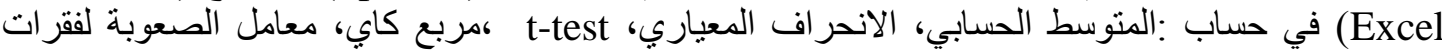



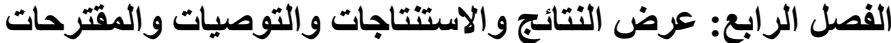

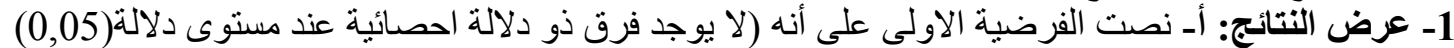

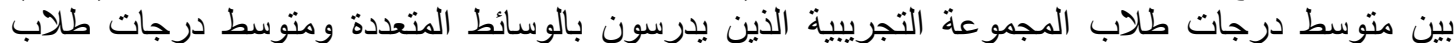


و الانحر اف المعياري والقيمة التائية لدرجات مجموعتي البحث في اختبار التحصيل، وكما موضح في الجدول

جدول (2) نتائج اختبار (t.test) لارجات مجموعتي البحث في اختبار التحصيل

\begin{tabular}{|c|c|c|c|c|c|c|c|}
\hline \multirow{2}{*}{ الدلالة الاحصائية عند (0,05) } & \multicolumn{2}{|c|}{ القيمة التائية } & \multirow{2}{*}{ الحرية } & \multirow{2}{*}{ الالتحراف } & \multirow{2}{*}{ الحستبي } & \multirow[t]{2}{*}{ عدد الطلاب } & \multirow[t]{2}{*}{ المجموعة } \\
\hline & الجدولية & المحسوبة & & & & & \\
\hline \multirow{2}{*}{ دالة } & \multirow[t]{2}{*}{2} & \multirow[t]{2}{*}{5,94} & \multirow[t]{2}{*}{58} & 5,92 & 40,48 & 30 & التجريبية \\
\hline & & & & 6,34 & 29,46 & 30 & الضابطة \\
\hline
\end{tabular}

يتضح من الجدول أعلاه أن القيمة التائية المحسوبة بلغت (5,94) و هي اكبر من القيمة الجدولية البالغة (2)



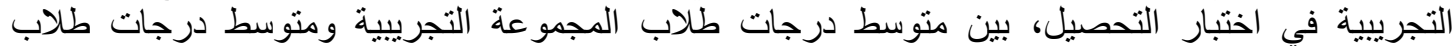


بـ نصت الفرضية الصفرية الثانية على أنه (لا يوجد فرق ذو دلالة احصائية عند مستوى دلالة (0,05) بين

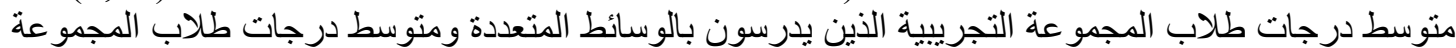

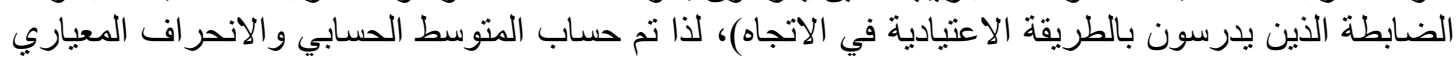


جدول (3) نتائج اختبار (t.test) لعينتين مستقلتين لمجموعتي البحث في مقياس الاتجاه

\begin{tabular}{|c|c|c|c|c|c|c|c|}
\hline \multirow{2}{*}{ الدلالة الاحصائية عند (0,05 ) } & \multicolumn{2}{|c|}{ القيمة التائية } & \multirow{2}{*}{ الحرية } & \multirow{2}{*}{ الالتحراف } & \multirow{2}{*}{ الحستوسط } & \multirow[t]{2}{*}{ عدد الطلاب } & \multirow[t]{2}{*}{ المجموعة } \\
\hline & الجدولية & المحسوبة & & & & & \\
\hline \multirow{2}{*}{ دالة } & \multirow[t]{2}{*}{2} & \multirow[t]{2}{*}{10,25} & \multirow[t]{2}{*}{58} & 4,30 & 31,26 & 30 & التجرييية \\
\hline & & & & 7,32 & 18,6 & 30 & الضابطة \\
\hline
\end{tabular}






Journal of Arts, Literature, Humanities and Social Sciences

ISSN online: 2414 - 3383

ISSN print: 2616 - 3810

ديسمبر 2019

Volume (46)

العدد (46)

December 2019

ويظهر من الجدول ان القيمة النائية المحسوبة بلغت (10,25)، وهي أكبر من القيمة الجدولية البالغة (2) عند درجة حرية (58) ومستوى دلالة (0,05) وهو ما يعني وجود فرق ذو دلالية الالة إحصائية لصالح المجموعة التجريية في مقياس الاتجاه .

Conclusions الاستنتاجات فئات فئان

في ضوء النتائج التي أسفر عنها البحث الحاليّ يمكن للباحث استنتاج الآتي:-


تحصيلهم الدر اسي مقارنةً بالطريقة الاعتيادية .

2. فاعلية التدريس بالوسائط المتعددة في الاتجاه نحو المادة مقارنةً بالطريقة الاعتيادية .

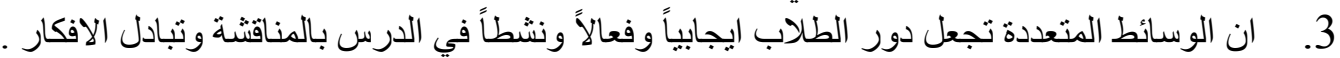


في ضوء النتائج التي أسفر عنها البحث، يوصدي الباحثان بالآتي :





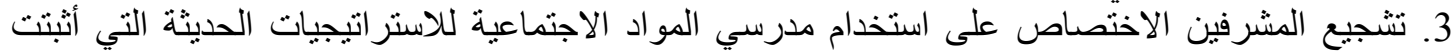

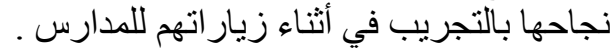

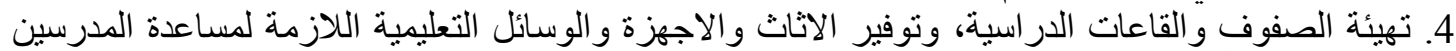



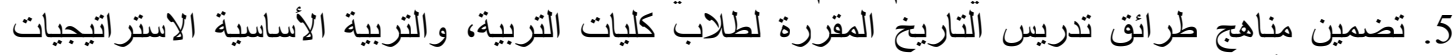
و الطر ائق و الأساليب الحديثة في العملية التدريسية ومنها استر اتيجيات الوسائط التئرة التعددة .

خامساً: المقترحات Proposals استكمالاً للبحث يقترح البات البثان عدة مقترحات وكما يلي: 1. دراسة فاعلية الوسائط المتعددة في متغيرات ألخات أخر مثل تنمية أنواع من التفكير كالتفكير العلمي، أو التفكير

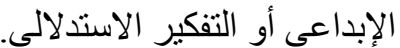
2. دراسة فاعلية الوسائط المتعددة في مر احل دراسية أخرى مثل المرحلة الإعدادية مع أخذ متغيرات أخرى بنظر الاعتبار. 3. إجر اء دراسات مماتلة للار اسة الحالية في مو اد در اسية اخرى كمادة الفيزياء أو الكيمياء أو الرياضيات.


المر احل المنتهية على أستر اتيجيات الوسائط المتعددة . لئل

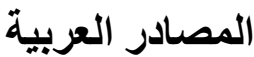

1. الحريري، رافدة (2016): الجودة الثاملة في المناهج وطرق التدريس ، ط2، دار المسيرة ، عمان.

2.



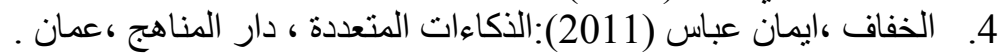

5.

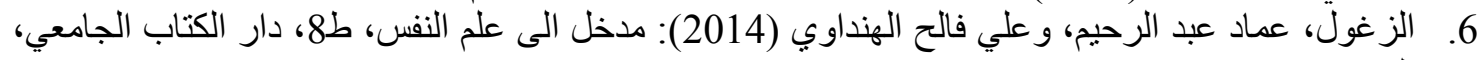



7. زيتون، كمال عبد الحميد (2002): تكنولوجيا التعليم في عصر المعلومات والاتصالات، عالم الكتب، القاهرة. 




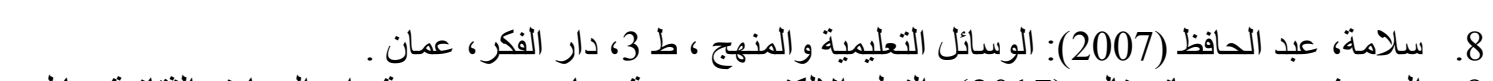

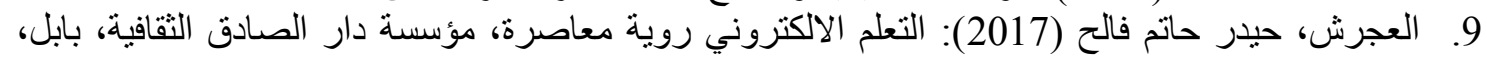

10. علام ، صعلاح الدين محمود(2011): القياس والتقويم التربوي في العملية التدريسية، ط4 ، دار المسيرة ،

عمان . عيلان

11. عيادات، يوسف (2004): الحاسوب التعليمي وتطبيقاته التربوية ، دار المسيرة، عمان.



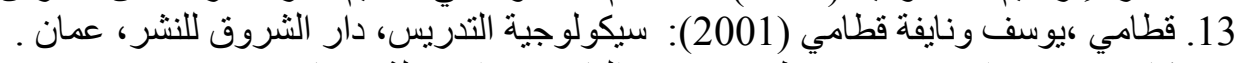

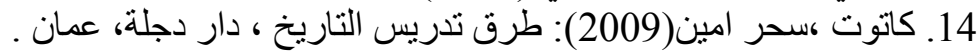









الامير للطباعة و الاستنساخ، بغداد.

\section{References}

1. Hariri, Rafida (2016): Total Quality in Curricula and Teaching Methods, Dar AlMasira, Amman.

2. Al-Khalidi, Adeeb (2001): Mental Health, Arab Publishing House, University Library, Gharyan, Libya.

3. Khader, Fakhri Rashid (2006): Methods of Teaching Social Studies, Dar AlMasira, Amman, Jordan.

4. Al-Khafaf, Iman Abbas (2011): Multiple Intelligences, Dar Al-Manahj, Amman.

5. Al-Rubaie, Mahmoud Daoud (2006): Contemporary Teaching Methods and Methods, Modern Book World, Amman.

6. Al-Zghoul, Emad Abdul Rahim, and Ali Faleh Al-Hindawi (2014): Introduction to Psychology, 8th Floor, University Book House, Al Ain, UAE.

7. Zeitoun, Kamal Abdel Hamid (2002): Educational Technology in the Information and Communication Era, Book World, Cairo.

8. Salameh, Abdul Hafiz (2007): Teaching Aids and Curriculum, Dar Al-Fikr, Amman.

9. Al-Ajrash, Haider Hatem Faleh (2017): E-learning a contemporary vision, Dar AlSadiq Cultural Foundation, Babylon, Iraq.

10. Allam, Salah Al-Din Mahmoud (2011): Educational Measurement and Evaluation in the Teaching Process, 4th Floor, Dar Al-Masira, Amman.

11. Clinics, Yousef (2004): Educational Computer and its Educational Applications, Dar Al-Masira, Amman.

12. Al-Far, Ibrahim Abdul-Wakil (2001): Using Computer in Education, Dar Al-Fikr, Amman, Jordan.

13. Qatami, Yusuf and Nayfeh Qatami (2001): The Psychology of Teaching, AlShorouk Publishing House, Amman.

14. Katot, Sahar Amin (2009): Methods of Teaching History, Dar Tigris, Amman. 


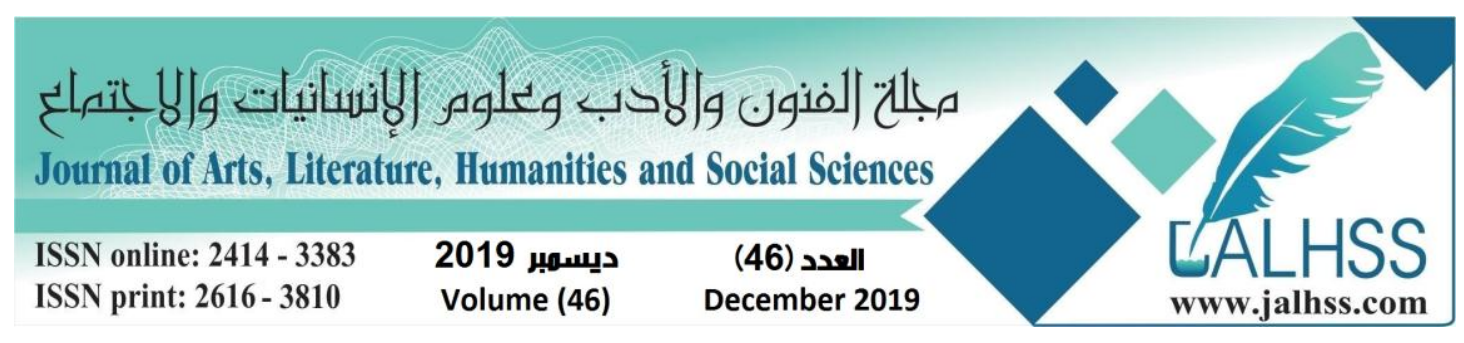

15. Mohammed, Mustafa Abdel Samie (2009): Computerized Children Programs, Dar Al Fikr, Amman.

16. Mohammed, Jassim Mohammed (2006) learning theories, House of Culture for publication and distribution, Amman.

17. Maaytah, Khalil Abdul Rahman (2000): Social Psychology, Dar Al-Fikr for Printing and Publishing.

18. Najm, Saadoun Salman, and Khulood Aziz Rahim (2016): Measurement and evaluation in education and psychology, Prince Printing and Copying Office, Baghdad.

19. Bedworth , David A\& Albert E. Bedworth(2010): The Dictionary of Health Education, Oxford University Press, New York(U.S.A).

20. Evans, Dennis A (2005): Taking Sides Clashing Views On Controler Sial, second Edition, Mc Graw Hill, Dushkin.

21. Everitt, B. S(2003): THE CAMBRIDGE DICTIONARY OF Statistics, Second Edition, Cambridge, New York(U.S.A).

22. Hazlina,Noor\& Kamaruzaman Jusoff,( 2009):Using multimedia in teaching Islamic studies, Journal Media and Communication Studies Vol. 1, No. (5) , p(86-94). 23. Plack, Joanna Miriam (2002): Teacher- student relationships An examination of digital multimedia teaching and learning, $\mathrm{PhD}$, University of Toronto, Canada.

24. Rani,T.Swarupa et .al (2007): Educational Measurement and Evaluation, 2nd Edition, Discovery Publishing House, Delhi, India .

25. Schultz, Fred (2008): Multicultural Education, 14Edition, Mc Grew Hill, New York (U. S.A).

26. Solomon , p . \& Sue , B (2005): Innovations In Rehabilitation Scienese Ducation , Berlin, Springer.

27. Vanden Bos (2015): APA Dictionary of Psychology, second Edition, American, Psychological Association, Washington (U.S.A).

28. Watkins, et al (2007): Effective Learning in Classrooms, First published, Paul Chapman Publishing, London . 